\title{
Higher descent data as a homotopy limit
}

\author{
Matan Prasma
}

Received: 9 March 2012 / Accepted: 5 July 2013 / Published online: 13 August 2013

(C) Tbilisi Centre for Mathematical Sciences 2013

\begin{abstract}
We define the 2-groupoid of descent data assigned to a cosimplicial 2-groupoid and present it as the homotopy limit of the cosimplicial space gotten after applying the 2-nerve in each cosimplicial degree. Our techniques apply to the case of $n$-groupoids as well thus providing an analogous presentation of "descent data" in higher dimensions.
\end{abstract}

Keywords Descent data $\cdot$ 2-groupoid $\cdot$ Homotopy limit

Mathematics Subject Classification $14-\mathrm{XX}$

\section{Introduction}

In this note we reinterpret algebro-geometric information, namely descent data, in a homotopically-invariant way. Given a cosimplicial 2-groupoid $\mathcal{G}^{\bullet}$, its descent data is (the 2-nerve of) a 2-groupoid $\operatorname{Desc}\left(\mathcal{G}^{\bullet}\right):=\operatorname{Tot}_{r}\left(\mathbb{N G} \mathcal{G}^{\bullet}\right)$ (where $\operatorname{Tot}_{r}$ means "totalization without degeneracies") whose path components coincide with the set of descent data modulo the gauge equivalence relation (see [2] and also [20, Definitions 1.4, 1.5]). We show that this 2-groupoid is (canonically equivalent to) the homotopy limit $\operatorname{holim}_{\triangle} \mathbb{N} \mathcal{G}^{\bullet}$ where $\mathbb{N}$ is the 2-nerve applied on each level. Thus, given a weak equivalence of cosimplicial 2-groupoids $\mathcal{G}^{\bullet} \rightarrow \mathcal{H}^{\bullet}$, the map $\operatorname{Desc}\left(\mathcal{G}^{\bullet}\right) \rightarrow \operatorname{Desc}\left(\mathcal{H}^{\bullet}\right)$ is a weak equivalence of 2-groupoids; this generalizes [20, Theorem 0.1]. We know of two situations in which this setup can arise.

Communicated by Ronald Brown.

M. Prasma $(\varangle)$

Mathematics Department, Radboud University, P.O. Box 9102, 6500 HC Nijmegen, Holland e-mail: matan.prezma@mail.huji.ac.il 
The first concerns Maurer-Cartan equations. Consider a cosimplicial DGLA, which shows up for instance as the Čech construction for a sheaf of nilpotent parameter DGLAs. Taking the Deligne 2-groupoid (which encodes solutions to Maurer-Cartan equations) of each cosimplicial degree gives rise to a cosimplicial 2-groupoid. As follows from [21, Theorem 0.4], a quasi-isomorphism of cosimplicial pronilpotent DGLAs of quantum type (i.e. concentrated in degrees $\geq-1$ ) induces a weak equivalence of cosimplicial 2-groupoids.

The second is in the classification of $\mathcal{G}$-gerbes for a sheaf of groups $\mathcal{G}$ (see $[5,6]$ ). There, the cosimplicial 2-groupoid arises via the Čech construction (with respect to a cover) from the sheaf of 2-groups (or crossed modules) $\mathcal{G} \rightarrow \operatorname{Aut}(\mathcal{G})$ and the descent data approximates isomorphism classes of $\mathcal{G}$-gerbes. In some cases, for example when the cover totally trivializes all $\mathcal{G}$-gerbes, $\pi_{0} \operatorname{Desc}(\mathcal{G})$ will classify all $\mathcal{G}$-gerbes and a refinement will yield a weak equivalence of cosimplicial 2-groups.

Descent data is intimately related to non-abelian cohomology. For this reason, the role of codegeneracies is degenerate and we can consider the restricted totalization (see Sect. 2) which simplifies the homotopical framework. This eliminates the difficulty arising from the fact that the cosimplicial simplicial set gotten by taking the 2-nerve of each level of a cosimplicial 2-groupoid need not be Reedy fibrant (see [13, Example 8]) and gives an argument which is also valid for the case of $n$-groupoids; this is discussed in Sect. 7.

\section{Totalization and restricted totalization}

Let $\Delta$ be the category whose objects are non-empty finite ordinals $[0],[1], \ldots,[n]$ where $[n]=\{0,1, \ldots, n\}$ and whose morphisms are weakly order preserving functions. Every morphism in $\Delta$ is a composition of face maps $d^{i}:[n-1] \rightarrow[n]$ and degeneracies $s^{i}:[n+1] \rightarrow[n], i=0, \ldots, n$. A simplicial set is a functor $X: \Delta^{o p} \rightarrow$ Set and we write $X_{n}:=X([n]), d_{i}:=X\left(d^{i}\right), s_{i}:=X\left(s^{i}\right)$. Write $s$ Set for the category whose objects are simplicial sets and whose morphisms are natural transformations.

A cosimplicial object in a category $\mathcal{C}$ is a functor $\Delta \rightarrow \mathcal{C}$. In particular, a cosimplicial simplicial set is a cosimplicial object in $s \mathcal{S} e t$. We write $s \mathcal{S} e t^{\triangle}$ for the category whose objects are cosimplicial simplicial sets and whose morphisms are natural transformations. If $X^{\bullet}$ is a cosimplicial object we will denote the object assigned to $[n]$ by $X^{n}$. The maps $d^{i}:=X\left(d^{i}\right)$ and $s^{i}:=X\left(s^{i}\right)$ are called cofaces and codegeneracies respectively. The cosimplicial standard simplex $\Delta^{\bullet}$ has $\Delta^{n}$ in its $n$-th cosimplicial degree and cofaces and codegeneracies induced by precomposition. For $X^{\bullet}, Y^{\bullet} \in s \mathcal{S} e t^{\Delta^{\natural}}$, the product $(X \times Y)^{\bullet}$ is the cosimplicial simplicial set with $(X \times Y)^{n}:=X^{n} \times Y^{n}$ and for $A \in s \mathcal{S}$ et, we write, by abuse of notation, $A$ for the constant cosimplicial simplicial set with $A^{n}:=A$ for all $n$ and cofaces and codegeneracies being identities.

The category $s \mathcal{S} e t^{\triangle}$ is enriched over simplicial sets. Given $X^{\bullet}, Y^{\bullet} \in s \mathcal{S e t}{ }^{\triangle}$, the 'internal hom' $\underline{s \mathcal{S e t}^{\triangle}}\left(X^{\bullet}, Y^{\bullet}\right)$ is the simplicial set whose $n$-simplices are

$$
\underline{s \mathcal{S e t}^{\triangle}}\left(X^{\bullet}, Y^{\bullet}\right)_{n}=\operatorname{sSet}^{\bowtie}\left(X^{\bullet} \times \Delta^{n}, Y^{\bullet}\right)
$$

Here, $X^{\bullet} \times \Delta^{n}$ is the product of $X^{\bullet}$ with the constant cosimplicial simplicial set $\Delta^{n}$. 
With this enrichment, $s \mathcal{S e t}{ }^{\triangle}$ is a simplicial category in the sense of [11, II,2.1] or in our terminology, tensored and cotensored over $s$ Set (see [11, II, 2.5]). For $A, B \in$ $s \mathcal{S} e t^{\triangle}$ we denote the tensor and cotensor functors by

$$
A \times(-): s \mathcal{S e t} \rightarrow s \mathcal{S} e t^{\triangle} \text { and } B^{(-)}:(s \mathcal{S} e t)^{o p} \rightarrow s \mathcal{S} e t^{\triangle}
$$

respectively; these are the left adjoints of $\underline{s \mathcal{S} e t^{\triangle}}(A,-)$ and $\underline{s \mathcal{S} e t^{\unrhd}}(-, B)$.

Definition 2.1 The totalization Tot $: s \mathcal{S e t}^{\triangle} \rightarrow s \mathcal{S}$ et is the simplicial set $\operatorname{Tot}\left(X^{\bullet}\right)=$ $\underline{\operatorname{Set}^{\triangle}}\left(\Delta^{\bullet}, X^{\bullet}\right)$.

We let $\Delta_{r}$ denote the subcategory of $\Delta$ with the same objects but only injective maps i.e. compositions of face maps $d^{i}$. A restricted cosimplicial object in a category $\mathcal{C}$ is a functor $\Delta_{r} \rightarrow \mathcal{C}$; it is also called a semi-cosimplicial object by some authors. In particular, a restricted cosimplicial object in $s \mathcal{S}$ et is called a restricted cosimplicial simplicial set. There is an obvious 'restriction' functor $r: s \mathcal{S e t}^{\Delta} \rightarrow s \mathcal{S e t}^{{ }^{\Delta_{r}}}$ and in particular we have $r \Delta^{\bullet} \in s \mathcal{S} e t^{\Delta_{r}}$. The category $s \mathcal{S} e t^{\Delta_{r}}$ is again enriched over

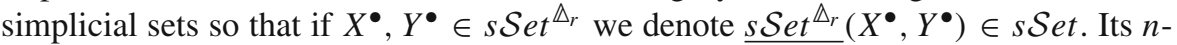
simplices are $\underline{s \mathcal{S e t}^{\Delta_{r}}}\left(X^{\bullet}, Y^{\bullet}\right)_{n}:=s \mathcal{S e t}^{\Delta_{r}}\left(X^{\bullet} \times r \Delta^{n}, Y^{\bullet}\right)$ and given $\theta:[m] \rightarrow[n]$

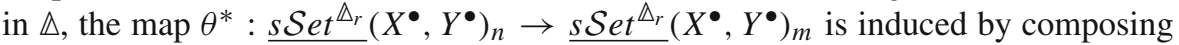
with the map $\theta_{*}: r \Delta^{m} \rightarrow r \Delta^{n}$. Simplicial identities hold since their opposites hold in $\Delta^{\bullet}$. The arguments in [11, II,2.5] may be used verbatim to show that $s \mathcal{S e t}^{\Delta_{r}}$ is tensored and cotensored over $s \mathcal{S}$ et.

Definition 2.2 The restricted totalization is the functor Tot $_{r}: s \mathcal{S} e t^{\Delta_{r}} \rightarrow s \mathcal{S e t}$ defined by $\operatorname{Tot}_{r}\left(X^{\bullet}\right)=\underline{s \mathcal{S e t}^{\Delta_{r}}}\left(r \Delta^{\bullet}, X^{\bullet}\right)$.

More generally we can use ends (see [14, IX.5]) to get:

Definition 2.3 Let $\mathcal{C}$ be a category cotensored over simplicial sets.

1. The totalization of $\mathcal{G}^{\bullet} \in \mathcal{C}^{\triangle}$ is the object of $\mathcal{C}$ is given by the end

$$
\operatorname{Tot}\left(\mathcal{G}^{\bullet}\right):=\int_{[n] \in \triangle}\left(\mathcal{G}^{n}\right)^{\Delta^{n}} .
$$

2. The restricted totalization of $\mathcal{G}^{\bullet} \in \mathcal{C}^{\triangle_{r}}$ is the object of $\mathcal{C}$ is given by the end

$$
\operatorname{Tot}_{r}\left(\mathcal{G}^{\bullet}\right):=\int_{[n] \in \triangle_{r}}\left(G^{n}\right)^{\Delta^{n}} .
$$

\section{Model structures}

We assume the reader is familiar with the definition of a model category. Let us shortly spell out the definition of a simplicial model category.

Definition 3.1 A model category $\mathcal{M}$ is called simplicial if it is enriched with tensor and cotensor over $s \mathcal{S}$ et and satisfies the following axiom [17, II.2 SM7]: If $f: A \rightarrow B$ 
is a cofibration in $\mathcal{M}$ and $i: K \rightarrow L$ is a cofibration in $s \mathcal{S e t}$ then the map

$$
q: A \otimes L \coprod_{A \otimes K} B \otimes K \rightarrow B \otimes L
$$

1. is a cofibration;

2. is a weak equivalence if either

(a) $f$ is a weak equivalence in $\mathcal{M}$ or

(b) $i$ is a weak equivalence in $s$ Set.

Definition 3.2 A category $\mathcal{R}$ is called a Reedy category if it has two subcategories $\mathcal{R}_{+}, \mathcal{R}_{-} \subseteq \mathcal{R}$ and a degree function $d: o b(\mathcal{R}) \rightarrow \alpha$ where $\alpha$ is an ordinal number such that:

- Every non-identity morphism in $\mathcal{R}_{+}$raises degree;

- Every non-identity morphism in $\mathcal{R}_{-}$lowers degree;

- Every morphism in $\mathcal{R}$ factors uniquely as a map in $\mathcal{R}_{-}$followed by a map in $\mathcal{R}_{+}$.

The category $\Delta$ is a Reedy category with $\Delta_{+}=\Delta_{i n j}\left(=\Delta_{r}\right), \Delta_{-}=\Delta_{\text {surj }}$ and the obvious degree function.

Let $\mathcal{R}$ be a Reedy category and $\mathcal{C}$ any category. Given a functor $X: \mathcal{R} \rightarrow \mathcal{C}$ and an object $n \in \mathcal{R}$ we set $X^{n}:=X(n)$ (to relate to the case $\mathcal{R}=\Delta$ ), and define the $n$-th latching object to be

$$
L^{n} X=\operatorname{colim}_{\llbracket(\mathcal{R})} X^{s}
$$

where $\mathbb{L}(\mathcal{R})$ is the full subcategory of the over category $\mathcal{R}_{+} / n$ containing all objects except the identity $i d_{n}$.

Dually, define the $n$-th matching object to be

$$
M^{n} X=\lim _{M(\mathcal{R})} X^{s}
$$

where $M(\mathcal{R})$ is the full subcategory of the under category $n / \mathcal{R}_{-}$containing all objects except $i d_{n}$. We have natural morphisms

$$
L^{n} X \rightarrow X^{n} \rightarrow M^{n} X
$$

The importance of a Reedy structure on $\mathcal{R}$ is due to the following:

Theorem 3.3 [18] Let $\mathcal{R}$ be a Reedy category and $\mathcal{M}$ a model category. The functor category $\mathcal{M}^{\mathcal{R}}$ admits a structure of a model category, called Reedy model structure in which a map $X \rightarrow Y$ is a

- Weak equivalence iff $X^{n} \rightarrow Y^{n}$ is a weak equivalence in $\mathcal{M}$ for every $n$.

- Cofibration iff the map $L^{n} Y \bigsqcup_{L^{n} X} X^{n} \rightarrow Y^{n}$ is a cofibration in $\mathcal{M}$ for every $n$.

- Fibration iff the map $X^{n} \rightarrow M^{n} X \times_{M^{n} Y} Y^{n}$ is a fibration in $\mathcal{M}$ for every $n$.

In particular, an object $X$ is 
- Fibrant iff $X^{n} \rightarrow M^{n} X$ is a fibration in $\mathcal{M}$ for every $n$.

- Cofibrant iff $L^{n} X \rightarrow X^{n}$ is a cofibration in $\mathcal{M}$ for every $n$.

Moreover [1, Theorem 4.7], if the model structure on $\mathcal{M}$ is simplicial, so is the Reedy model structure on $\mathcal{M}^{\mathcal{R}}$.

Corollary 3.4 The Kan-Quillen model structure $s \mathcal{S e t}_{K-Q}$ and the Reedy structure on $\triangle$ (respectively $\Delta_{r}$ ) induce a simplicial model structure on $s \mathcal{S e t}^{\triangle}$ (respectively $\left.s \mathcal{S e t}^{\Delta_{r}}\right)$.

Example 1 The object $X=\Delta^{\bullet} \in s \mathcal{S} e t^{\triangle}$ is Reedy cofibrant. The map $L^{n} X \rightarrow X^{n}$ is the inclusion $\partial \Delta^{n} \hookrightarrow \Delta^{n}$ which is a cofibration of simplicial sets.

Next, we recall another model structure on $s \mathcal{S} e t^{\Delta_{r}}$.

Theorem 3.5 The simplicial enrichment of $s \mathcal{S e t}^{\Delta_{r}}$ can be extended to a simplicial model structure, called the projective model structure, in which a map $X \rightarrow Y$ is a

- weak equivalence if for each $n, X^{n} \rightarrow Y^{n}$ is a weak equivalence.

- fibration if for each $n, X^{n} \rightarrow Y^{n}$ is a Kan fibration.

- cofibration if it has the left lifting property with respect to trivial fibrations.

In particular, $X$ is a fibrant object iff $X^{n}$ is a Kan complex for every $n$.

Suppose $\mathcal{R}$ is a Reedy category and $\mathcal{M}$ is a model category. In general, if the projective model structure on $\mathcal{M}^{\mathcal{R}}$ exists (e.g. when $\mathcal{M}$ is sufficiently nice) it will be very different than the Reedy model structure. However, in special cases the two may coincide.

Proposition 3.6 If $\mathcal{R}=\mathcal{R}_{+}$the projective and Reedy model structures on $\mathcal{M}^{\mathcal{R}}$ coincide.

Proof In this case, for every $X \in \mathcal{M}$ the $n$-th matching object $M^{n} X$ equal the terminal object, being the limit over the empty diagram, so that a map $X \rightarrow Y$ is a Reedy fibration iff $X^{n} \rightarrow Y^{n}$ is a fibration in $\mathcal{M}$. This means that the two model structures have the same classes of weak equivalences and fibrations, and hence coincide.

For $\mathcal{R}=\Delta_{r}$ we obtain:

Corollary 3.7 The Reedy and projective model structures on $s$ Set ${ }^{\Delta_{r}}$ coincide. Thus, an object $X \in s \mathcal{S e t}^{{ }^{\Delta_{r}}}$ is Reedy fibrant iff $X^{n}$ is a Kan complex for each $n$.

Remark 3.8 By Example 1, $\Delta^{\bullet}$ is Reedy cofibrant in $s \mathcal{S} e t^{\triangle}$ and since the indexing category defining $L^{n} \Delta^{\bullet}$ depends only on $\Delta_{+}=\Delta_{r}$, we have $L^{n} \Delta^{\bullet}=L^{n} r \Delta^{\bullet}$. Thus, the map $L^{n} r \Delta^{n} \rightarrow r \Delta^{n}$ is again the inclusion $\partial \Delta^{n} \hookrightarrow \Delta^{n}$ so that $r \Delta^{\bullet}$ is Reedy cofibrant in $s$ Set ${ }^{\Delta_{r}}$.

\section{2-Groupoids}

Definition 4.1 A (strict) 2-groupoid is a groupoid-enriched (small) category in which all morphisms are invertible.

Explicitly, a 2-groupoid consists of: 
- a set of objects;

- for every pair of objects $x, y$, a set of 1-morphisms, written as $f: x \rightarrow y$; and, for every object $x$, a distinguished 1-morphism $1_{x}: x \rightarrow x$;

- for every pair of 1-morphisms $f, g: x \rightarrow y$ a set of 2-morphisms, written as $a: f \Rightarrow g$; and, for every 1-morphism $f$, a distinguished 2-morphism $1_{f}: f \Rightarrow f$

together with a composition law for 1-morphisms and vertical and horizontal composition laws for 2-morphisms (denoted by $*$ and o respectively) subject to three axioms, expressing associativity of composition and left and right unit laws and in addition satisfy the 'interchange law':

$$
(b * a) \circ\left(b^{\prime} * a^{\prime}\right)=\left(b^{\prime} \circ b\right) *\left(a^{\prime} \circ a\right) .
$$

All morphisms are invertible with respect to these composition laws.

There are 2-categorical analogues for the notions of a functor and natural transformation. However, since 2-categories have 2-morphisms, an additional 'level of arrows' reveals itself, namely, the one of modifications. There is some ambiguity regarding these notions, since one can consider also their weak versions. For the sake of clarity, we spell out the definitions we use, which are taken from [10, I,2.2;I,2.3].

Definition 4.2 Let $\mathcal{G}, \mathcal{H}$ be a pair of 2-groupoids.

(I) A (strict) 2-functor $\Phi: \mathcal{G} \rightarrow \mathcal{H}$ is a groupoid-enriched functor between the underlying groupoids of $\mathcal{G}$ and $\mathcal{H}$. Explicitly, $\Phi$ assigns:

- to each object $x \in \mathcal{G}$, an object $\Phi x \in \mathcal{H}$,

- to each 1-morphism $f: x \rightarrow y \in \mathcal{G}$, a 1-morphism $\Phi f: \Phi x \rightarrow \Phi y \in \mathcal{H}$,

- to each 2-morphism $a: f \Rightarrow g \in \mathcal{G}$ a 2-morphism $\Phi a: \Phi f \Rightarrow \Phi g \in \mathcal{H}$

and this assignment respects all compositions and units.

(II) Given a pair of 2-functors $\Phi, \Psi: \mathcal{G} \rightarrow \mathcal{H}$ between 2-groupoids, a (strict) 2natural transformation $\Theta: \Phi \Rightarrow \Psi$ consists of a 1-morphism $\eta_{x}: \Phi x \Rightarrow \Psi x$ for every object $x \in \mathcal{G}$ which is natural in the sense that for every 2-morphism $a: f \Rightarrow g$ in $\mathcal{G}$, the diagram

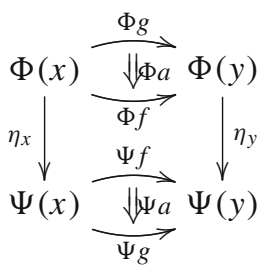

is commutative in that $1_{\eta_{y}} \circ \Phi a=\Psi a \circ \eta_{x}$ as 2-morphisms in $\mathcal{H}$.

(III) Given a pair of 2-natural transformations $\eta, \theta: \Phi \Rightarrow \Psi$, a modification $\mu: \eta \Rightarrow$ $\theta$ consists of a 2-morphism $\mu_{x}: \eta_{x} \Rightarrow \theta_{x}$ in $\mathcal{H}$ for every object $x \in \mathcal{G}$ such that for every 1-morphism $f: x \rightarrow y$ in $\mathcal{G}$, the diagram 


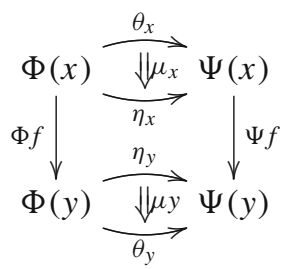

is commutative in the sense of (II).

We denote by $2 \mathcal{G} p d$ the category of 2 -groupoids and strict 2-functors between them. The collection of 2-functors from $\mathcal{G}$ to $\mathcal{H}$, their 2-natural transformations and their modifications is naturally a 2-category (see [10, 2.3]) which is in fact a 2-groupoid because of invertibility of 1-and 2-morphisms in the codomain $\mathcal{H}$. We denote this 2-groupoid by $2 \mathcal{G} p d(\mathcal{G}, \mathcal{H})$.

Theorem 4.3 (cf. [10, 2.3]) The category $2 \mathcal{G} p d$ is cartesian closed with respect to $2 \mathcal{G} \operatorname{pd}(\mathcal{G}, \mathcal{H})$.

Let $\Delta_{\leq n}$ be the full subcategory of $\Delta$ with objects [0], ., [n] and let $s \mathcal{S e t}_{\leq n}$ be the category of functors $\left(\Delta_{\leq n}\right)^{o p} \rightarrow \mathcal{S} e t$. Objects of $s$ Set $\leq n$ are called $n$-truncated

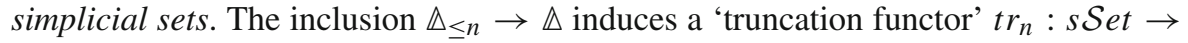
$s \mathcal{S e t}_{\leq n}$ which admits right and left adjoints $\cos _{n}: s \mathcal{S e t}_{\leq n} \rightarrow s \mathcal{S e t}$ and $s k_{n}$ : $s \mathcal{S e t}_{\leq n} \rightarrow s \mathcal{S}$ et respectively. We denote by $\operatorname{Cos}_{n}: s \mathcal{S} e t \rightarrow s \mathcal{S} e t$ the composition $\cos k_{n} \circ t r_{n}$ and by $S k_{n}$ the composition $s k_{n} \circ t r_{n}$. The functor $S k_{n}$ takes a simplicial set and creates a new simplicial set from its $n$-truncation by adding degenerate simplices in all levels above $n$; it is the simplicial analogue of the $n$-skeleton of a $C W$ complex. The functor $\operatorname{Cos}_{n}$ has a more involved simplicial description; it is the simplicial analogue of the $(n-1)$ th Postnikov piece $P_{n-1}$.

By abstract considerations, one can show that $\operatorname{Cos}_{n}$ is right adjoint to $S k_{n}$. Thus, a map $X \rightarrow \operatorname{Cos}_{n} Y$ corresponds precisely to a map $S k_{n} X \rightarrow Y$.

Definition 4.4 A simplicial set $X$ is called $n$-coskeletal if the canonical map $X \rightarrow$ $\operatorname{Cosk}_{n} X$ is an isomorphism.

In particular, given an $n$-truncated simplicial set $X, \cos _{n} X$ is an $n$-coskeletal simplicial set. Thus, in order to define an $n$-coskeletal simplicial set it is enough to define its $n$-truncation.

In Sect. 5 we intend to interpret the definition of descent data in terms of the 2nerve. In order to improve readability, we now rewrite the definition of $[15, \S 2]$ with the notations relevant for our formulae.

Definition 4.5 The 2-nerve is the functor $\mathbb{N}: 2 \mathcal{G} p d \rightarrow s \mathcal{S}$ et which takes a 2-groupoid $\mathcal{G}$ to the 3-coskeletal simplicial set $\mathbb{N G}$ whose

- 0 -simplices are the objects of $\mathcal{G}$;

- 1-simplices are the morphisms of $\mathcal{G}$;

- 2-simplices are triangles of the form 


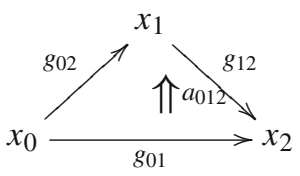

where $g_{i j}: x_{i} \rightarrow x_{j}$ and $\alpha: g_{02} \Rightarrow g_{12} \circ g_{01}$ are 1-and 2-morphisms (respectively) in $\mathcal{G}$;

- 3-simplices are commutative tetrahedra of the form

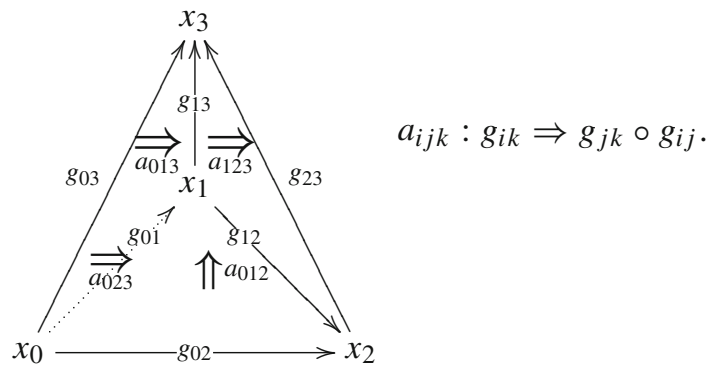

Commutativity of this tetrahedron means that the diagram of 2-morphisms

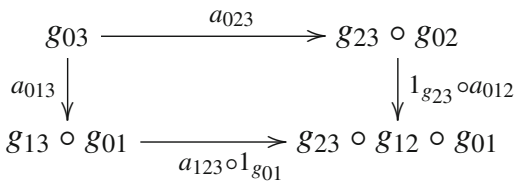

commutes.

We will need four well-known properties of the 2-nerve:

\section{Proposition 4.6 [15]}

1. N preserves products.

2. For every 2-groupoid $\mathcal{G}, \mathbb{N G}$ is a Kan complex.

3. A map of 2-groupoids $\mathcal{G} \rightarrow \mathcal{H}$ is a weak equivalence iff $\mathbb{N G} \rightarrow \mathbb{N H}$ is a weak equivalence of simplicial sets.

4. $\mathbb{N}$ admits a left adjoint $\mathbb{W}: s \mathcal{S}$ et $\rightarrow 2 \mathcal{G}$ pd, called the Whitehead 2-groupoid.

The category $2 \mathcal{G} p d$ admits a natural simplicial enrichment via $\mathbb{N} 2 \mathcal{G} p d(-,-)$. This enrichment is nicely behaved in the following sense:

Proposition 4.7 The simplicially-enriched category $2 \mathcal{G}$ pd is tensored and cotensored over $s$ Set. 
Proof We need to verify the conditions of [11, II,2.1]. The functor $((-) \times \mathcal{G}) \circ \mathbb{W}$ is a left adjoint to $\mathbb{N} 2 \mathcal{G} p d(\mathcal{G},-)$ and the functor $2 \mathcal{G} p d(W(-), \mathcal{H})$ is a left adjoint to $\mathfrak{N} 2 \mathcal{G} p d(-, \mathcal{H})$.

Remark 4.8 It is worth notice that $[16,5.1]$ shows the inner hom described in 4.2 does not induce a simplicial model category structure on $2 \mathcal{G} p d$ via setting the simplicial mapping space to be $\mathbb{N} 2 \mathcal{G} p d(\mathcal{G}, \mathcal{H})$. However in the current note, the main homotopical part is done in the category of (cosimplicial) simplicial sets so that we do not need a full-fledged homotopy theory of $2 \mathcal{G} p d$.

We shall need a slight generalization of Proposition 4.7:

Proposition 4.9 IfC is tensored and cotensored over s $\mathcal{S}$ et and I is any small category, then the functor category $\mathcal{C}^{I}$ is again tensored and cotensored over s $\mathcal{S}$ et.

Proof Denote the inner homs and their adjoints by

$$
\mathcal{C} \underset{\underline{\mathcal{C}}(X,-)}{\stackrel{X \otimes(-)}{\rightleftarrows}} \text { Set } \quad \text { and } \quad \mathcal{C}^{o p} \underset{\underline{\mathcal{C}}(-, Y)}{\stackrel{Y^{(-)}}{\rightleftarrows}} \text { S Set }
$$

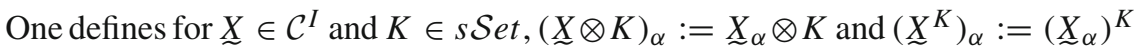
for every $\alpha \in I$. Then, $\underline{\mathcal{C}}^{I}(\underset{\sim}{X}, \underset{\sim}{Y})_{n}=\mathcal{C}^{I}\left(\underset{\sim}{X} \otimes \Delta^{n}, \underset{\sim}{Y}\right)$ with the obvious face and degeneracy maps provides the desired inner hom.

Corollary 4.10 The categories $2 \mathcal{G} p d^{\triangle}$ and $2 \mathcal{G} p d^{\triangle_{r}}$ are tensored and cotensored over simplicial sets.

The last corollary enables us to express the totalization as an end via Definition 2.3.

By abuse of notations, we denote by $\mathbb{N}, \mathbb{W}$ the prolongation of the 2-nerve and Whitehead 2-groupoid functors to the categories $2 \mathcal{G} p d^{\triangle}, s \mathcal{S} e t^{\triangle}$ (respectively). Since (level-wise) coproducts define the tensoring (over $s \mathcal{S} e t$ ) in $2 \mathcal{G} p d$ and $s \mathcal{S} e t^{\triangle}$ and $\mathbb{W}$ commutes with coproducts, the premisses of [11, Lemma 2.9(1)] are satisfied and we have:

Proposition 4.11 There is an enriched adjunction

$$
\underline{2 \mathcal{G} p d^{\triangle}}\left(\mathbb{W} \mathcal{G}^{\bullet}, \mathcal{H}^{\bullet}\right) \cong \underline{s \mathcal{S} e t^{\bowtie}}\left(\mathcal{G}^{\bullet}, \mathbb{N H}^{\bullet}\right)
$$

\section{Descent data of cosimplicial 2-groupoids}

Following [2], descent data of a cosimplicial crossed groupoid is defined in [20]. Since crossed groupoids can be viewed precisely as 2-groupoids (e.g. as a special case of [3]), a translation leads to the following:

Definition 5.1 (cf. [20, Definition 1.4]) Given a cosimplicial 2-groupoid $\mathcal{G}^{\bullet}=\left\{\mathcal{G}^{n}\right\}$, a descent datum is a triple $(x, g, a)$ in which:

1. $x$ is an object of $\mathcal{G}^{0}$; 
2. $g: d^{1} x \rightarrow d^{0} x$ is a 1-morphism in $\mathcal{G}^{1}$ and

3. $a: d^{1} g \Rightarrow d^{0} g \circ d^{2} g$ is a 2-morphism in $\mathcal{G}^{2}$.

such that

$$
\left(1_{d^{1} d^{0} g} \circ d^{3} a\right) * d^{1} a=\left(d^{0} a \circ 1_{d^{2} d^{2} g}\right) * d^{2} a . \quad(\text { twisted } 2-\text { cocycle) }
$$

Let $(x, g, a)$ be a descent datum of $\mathcal{G}^{\bullet}$.

Write $x_{i} \equiv x_{i}^{(1)}(i=0,1)$ for the object of $G^{1}$ corresponding to the vertex $(i)$ of $\Delta^{1}$, i.e. $x_{i}=d^{j} x$ where $\{j\}=\{0,1\} \backslash\{i\}$; thus $g: x_{0} \rightarrow x_{1}$.

Similarly, write $x_{i} \equiv x_{i}^{(2)}(i=0,1,2)$ and $g_{i j} \equiv g_{i j}^{(2)}(0 \leq i<j \leq 2)$ for (respectively) the object and 1-morphism of $\mathcal{G}^{2}$ corresponding to the vertex $(i)$ and edge $(i j)$ of $\Delta^{2}$. In other words, $x_{i}=d^{k} d^{j} x$ where $\{j<k\}=\{0,1,2\} \backslash\{i\}$ and $g_{i j}=d^{k} g$ where $\{k\}=\{0,1,2\} \backslash\{i, j\}$; thus $g_{i j}: x_{i} \rightarrow x_{j}$ and $a: g_{02} \Rightarrow g_{12} \circ g_{01}$.

Finally, write $x_{i} \equiv x_{i}^{(3)}(i=0, \ldots, 3), g_{i j} \equiv g_{i j}^{(3)}(i<j)$ and $a_{i j k} \equiv a_{i j k}^{(3)}(i<$ $j<k$ ) for (respectively) the object, 1-morphism and 2-morphism of $\mathcal{G}^{3}$ corresponding to the vertex $(i)$, edge $(i j)$ and face $(i j k)$ of $\Delta^{2}$; thus $g_{i j}: x_{i} \rightarrow x_{j}$ and $a_{i j k}: g_{i k} \Rightarrow$ $g_{j k} \circ g_{i j}$.

With these notations in mind, one can immediately see that the twisted cocycle condition corresponds precisely to the commutativity of a tetrahedron $t$ in $\mathcal{G}^{3}$ as in 4.5. Thus, such triples are in 1-1 correspondence with diagrams of simplicial sets of the form

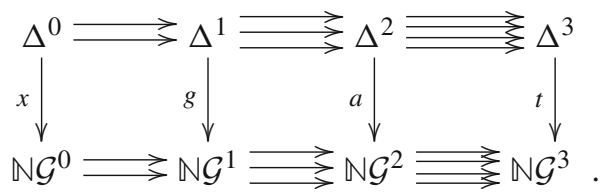

Since $\mathbb{N G}^{n}$ is 3-coskeletal, diagrams as above are in turn the 0 -simplices

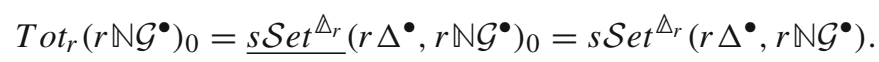

Definition 5.2 (cf. [20, definition 1.5]) Let $\mathbf{d}=(x, g, a), \mathbf{d}^{\prime}=\left(x^{\prime}, g^{\prime}, a^{\prime}\right)$ be a pair of descent data of $\mathcal{G}^{\bullet}$. A gauge transformation $\mathbf{d} \rightsquigarrow \mathbf{d}^{\prime}$ is a pair $(f, c)$ in which:

1. $f: x \rightarrow x^{\prime}$ is a 1-morphism in $\mathcal{G}^{0}$ and

2. $c: d^{0} f \circ g_{01} \Rightarrow g_{01}^{\prime} \circ d^{1} f$ is a 2-morphism in $\mathcal{G}^{1}$ (see Diagram 3)

$$
\underset{f_{0}}{f_{0} \underset{g_{01}^{\prime}}{\stackrel{g_{01}}{\longrightarrow}} x_{x_{1}^{\prime}} x_{1} f_{1} \quad\left(f_{0}:=d^{1} f, f_{1}:=d^{0} f\right)}
$$

such that the prism in $\mathcal{G}^{2}$ 


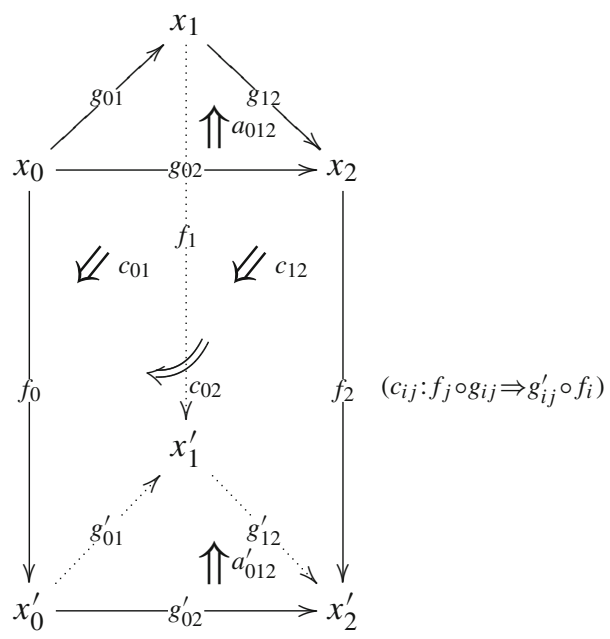

is commutative in the sense of 1 .

Let $\operatorname{Desc}\left(\mathcal{G}^{\bullet}\right)$ denote the set of descent data of $\mathcal{G}^{\bullet}$. The relation $\mathbf{d} R \mathbf{d}^{\prime} \Leftrightarrow \exists \mathbf{d} \rightsquigarrow \mathbf{d}^{\prime}$ is an equivalence relation on $\operatorname{Desc}\left(\mathcal{G}^{\bullet}\right)$ and we denote by $\overline{\operatorname{Desc}}\left(\mathcal{G}^{\bullet}\right)$ its quotient (cf. [20], definition 1.8). We now claim that:

Theorem 5.3 For any cosimplicial 2-groupoid $\mathcal{G}^{\bullet}$, there is a (natural) isomorphism

$$
\overline{\operatorname{Desc}}\left(\mathcal{G}^{\bullet}\right) \cong \pi_{0} \operatorname{Tot}_{r}\left(r \mathbb{N G}^{\bullet}\right)
$$

In order to prove Theorem 5.3 we would like to view a gauge transformation $\mathbf{d} \rightsquigarrow \mathbf{d}^{\prime}$ as a path between two vertices of $\operatorname{Tot}_{r}\left(r \mathbb{N} \mathcal{G}^{\bullet}\right)$ but there is a slight problem. Given a pair of descent data, thought of as 4-tuples $\mathbf{d}=(x, g, a, t)$ and $\mathbf{d}^{\prime}=\left(x^{\prime}, g^{\prime}, a^{\prime}, t^{\prime}\right)$ of the form 2, a path between them is an element of

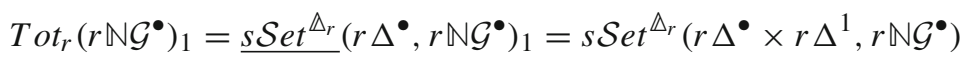

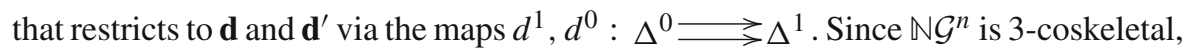
such elements correspond to diagrams of the form

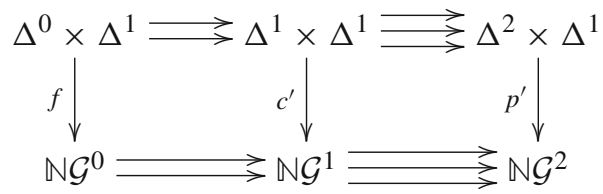

that restrict to $(x, g, a)$ and $(x, g, a)$. 
The last diagram carries an automatic 'triangulation'. The map $c^{\prime}$ is a diagram in $\mathcal{G}^{1}$ of the form

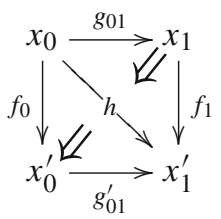

which is a triangulation of 3 ; and similarly, the map $p^{\prime}$ is a diagram in $\mathcal{G}^{2}$ which is a triangulation of 4 .

There are two possible solutions for that. The first (which was suggested by the referee) is to change the framework into crossed complexes, relying on [4, Theorem 2.4] and obtain a description of gauge transformations as maps of crossed complexes. The second, which we will adopt for the sake of simplicity, is to notice the following:

Lemma 5.4 Every gauge transformation $\mathbf{d} \rightsquigarrow \mathbf{d}^{\prime}$ gives rise to a canonical path in $\operatorname{Tot}_{r}\left(\mathbb{N G}^{\bullet}\right)$ between $\mathbf{d}$ and $\mathbf{d}^{\prime}$ and every such path gives rise to a canonical gauge transformation.

Proof Given a path between $\mathbf{d}$ and $\mathbf{d}^{\prime}$, represented by a triple $\left(f, c^{\prime}, p^{\prime}\right)$ as in 5, one can compose the 2-morphisms appearing in $c^{\prime}$ and in the squares of $p^{\prime}$ to obtain a triple $(f, c, p)$ and hence a gauge transformation $\mathbf{d} \rightsquigarrow \mathbf{d}^{\prime}$. Conversely, given a gauge transformation $(f, c): \mathbf{d} \rightsquigarrow \mathbf{d}^{\prime}$, one obtains, from condition 4 of Definition 5.2 a prism $p$ in $\mathcal{G}^{2}$. Then, by inserting the 1-morphism $f_{1} \circ g_{01}$ as the diagonal in 3 and $1_{f_{1} \circ g_{01}}$ in the upper triangle, one obtains a diagram of the form 6 and a similar procedure on 4 yields a prism $p^{\prime}$. The triple $\left(f, c^{\prime}, p^{\prime}\right)$ is the resulting path.

Expressing the totalization as an end allow us to reveal its higher structure:

Proposition 5.5 For a cosimplicial 2-groupoid $\mathcal{G}^{\bullet}$, there are natural isomorphisms

1. $\operatorname{Tot}\left(\mathbb{N G}^{\bullet}\right) \cong \mathbb{N} \operatorname{Tot}\left(\mathcal{G}^{\bullet}\right)$;

2. $\operatorname{Tot}_{r}\left(r \mathbb{N G}^{\bullet}\right) \cong \mathbb{N} \operatorname{Tot}_{r}\left(r \mathcal{G}^{\bullet}\right)$;

(see Definition 2.3).

Proof We only prove (1) as the proof of (2) is identical. Since $\mathbb{N}$ is a right adjoint, it commutes with limits. Relying on [14, IX.5],

$$
\mathbb{N} \operatorname{Tot}\left(\mathcal{G}^{\bullet}\right)=N\left(\int_{[n] \in \Delta}\left(\mathcal{G}^{n}\right)^{\Delta^{n}}\right) \cong \int_{[n] \in \triangle} \mathbb{N}\left(\left(\mathcal{G}^{n}\right)^{\Delta^{n}}\right) \cong \int_{[n] \in \Delta}\left(\mathbb{N} \mathcal{G}^{n}\right)^{\Delta^{n}}=\operatorname{Tot}\left(\mathbb{N} \mathcal{G}^{\bullet}\right)
$$

where the last isomorphism comes from [11, II, Lemma 2.9(2)] relying on the fact that $\mathbb{W}$ commutes with arbitrary coproducts. 
Thus, we define:

Definition 5.6 Given a cosimplicial 2-groupoid $\mathcal{G}^{\bullet}$, its descent 2-groupoid is $\operatorname{Desc}\left(\mathcal{G}^{\bullet}\right):=\operatorname{Tot}_{r}\left(\mathbb{N G}^{\bullet}\right)$.

Proof of Theorem 5.3 Since $\operatorname{Tot}_{r}\left(r \mathbb{N G} \mathcal{G}^{\bullet}\right)$ is a Kan complex (being the 2-nerve of a 2-groupoid), $\pi_{0} \operatorname{Tot}_{r}\left(\mathbb{N G} \mathcal{G}^{\bullet}\right)=\operatorname{Tot}_{r}\left(\mathbb{N G} \mathcal{G}^{\bullet}\right)_{0} / \sim$ where $\mathbf{d} \sim \mathbf{d}^{\prime}$ iff there is a path between them. By lemma 5.4 this equivalence relation is equal to the gauge equivalence relation.

\section{Invariance of descent data}

Theorem 5.3 enables us to use homotopy-theoretic tools to prove invariance of descent data under weak equivalence. We need one more simple theorem:

Theorem 6.1 For any cosimplicial 2-groupoid $\mathcal{G}^{\bullet}$, there is a (natural) weak equivalence $\operatorname{Tot}_{r}\left(\mathbb{N G}^{\bullet}\right) \simeq \operatorname{holim}_{\triangle} \mathbb{N} \mathcal{G}^{\bullet}$,

Proof In the simplicial model category $s \mathcal{S} e t^{\Delta_{r}}$ proj, the homotopy limit (over $\triangle_{r}$ ) of a fibrant object can be described as the internal mapping space from a weakly contractible cofibrant object [12, Theorem 19.4.6(2)]. In our case, $\mathbb{N G}^{\bullet}$ is fibrant and

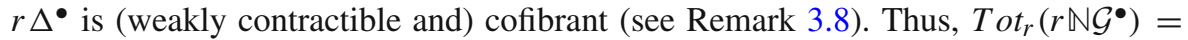
$\underline{s \mathcal{S e t}^{\Delta_{r}}}\left(r \Delta^{\bullet}, \mathbb{N G}^{\bullet}\right) \simeq \operatorname{holim}_{\triangle_{r}} r \mathbb{N} \mathcal{G}^{\bullet}$. By [9, Lemma 3.8], holim ${\triangle_{r}}_{r} \mathbb{N G}^{\bullet} \sim$ $\overline{\operatorname{holim}_{\triangle}} \mathbb{N} \mathcal{G}^{\bullet}$.

In light of Definition 5.6 and the previous theorem it now follows that:

Corollary 6.2 A weak equivalence of cosimplicial 2-groupoids $\mathcal{G}^{\bullet} \rightarrow \mathcal{H}^{\bullet}$ induces a weak equivalence of 2-groupoids $\operatorname{Desc}\left(\mathcal{G}^{\bullet}\right) \rightarrow \operatorname{Desc}\left(\mathcal{H}^{\bullet}\right)$.

In particular, we have:

Corollary 6.3 (cf. [20, Theorem 2.4]) If $\mathcal{G}^{\bullet} \rightarrow \mathcal{H}^{\bullet}$ is a weak equivalence of cosimplicial 2-groupoids, the induced map $\overline{\operatorname{Desc}}\left(\mathcal{G}^{\bullet}\right) \rightarrow \overline{\operatorname{Desc}}\left(\mathcal{H}^{\bullet}\right)$ is an isomorphism of sets

Proof By Theorems 5.3 and 6.1, the map $\overline{\operatorname{Desc}}\left(\mathcal{G}^{\bullet}\right) \rightarrow \overline{\operatorname{Desc}}\left(\mathcal{H}^{\bullet}\right)$ coincides with $\pi_{0}\left(\right.$ holim $\left._{\triangle} \mathbb{N G}^{\bullet}\right) \rightarrow \pi_{0}\left(\right.$ holim $\left._{\triangle} \mathbb{N H}^{\bullet}\right)$ and $\mathbb{N}$ and holim $\triangle$ preserve weak equivalences.

\section{Descent data of cosimplicial $\boldsymbol{n}$-groupoids}

The techniques of Sects. 4-6 work equaly well in higher dimensions. Here, we write down the details for the case of (strict) $n$-groupoids and the corresponding $n$-nerve $\mathbb{N}_{(n)}$ in the sense of [19] but the same arguments work for weaker notions of $n$-groupoids, e.g. Tamsamani $n$-groupoids. We only need two ingredients. The first is that $N_{(n)}$ admits a left adjoint (and hence commutes with limits); this is true since the inclusion $n \mathcal{G} p d \hookrightarrow n C a t$ admits a left adjoint $\Pi_{n}: n C a t \rightarrow n \mathcal{G} p d$ and thus the composite 
$\Pi_{n} \circ \tau_{n}$ (where $\tau_{n}$ is the fundamental $n$-category) is the desired left adjoint. The second ingredient is that $\mathbb{N}_{(n)} \mathcal{G}$ is a Kan complex for every $n$-groupoid $\mathcal{G}$; this goes back to [8].

In light of Theorem 5.3, it makes sense to define:

Definition 7.1 Let $\mathcal{G}^{\bullet}$ be a cosimplicial $n$-groupoid. Its $n$-descent data is the simplicial set $\operatorname{Desc}_{n}\left(\mathcal{G}^{\bullet}\right):=\operatorname{Tot}_{r}\left(\mathbb{N}_{(n)} \mathcal{G}\right)$.

Definition 7.1 makes sense formally, but its geometric meaning is unknown to us. Nevertheless, the formal reasoning of Proposition 5.5 implies:

Proposition 7.2 The simplicial set $\operatorname{Desc}_{n}\left(\mathcal{G}^{\bullet}\right)$ is the n-nerve of an n-groupoid.

Moreover, Theorem 6.1 generalizes immediately.

Theorem 7.3 Let $\mathcal{G}^{\bullet}$ be a cosimplicial (strict) n-groupoid. There is a natural weak equivalence $\operatorname{Tot}_{r}\left(r \mathbb{N}_{(n)} \mathcal{G}^{\bullet}\right) \simeq \operatorname{holim}_{\triangle} \mathbb{N}_{(n)} \mathcal{G}^{\bullet}$.

Acknowledgments I would like to thank Amnon Yekutieli for introducing and motivating the question at hand and to Yonatan Harpaz for a useful discussion. Thanks are extended to the referee for helpful remarks.

\section{References}

1. Angeltveit, V.: Enriched Reedy categories. Proc. Am. Math. Soc. 136(7), 2323-2332 (2008)

2. Bressler, P., Gorokhovsky, A., Nest, R., Tsygan, B.: Deformation quantization of gerbes. Adv. Math. 214(1), 230-266 (2007)

3. Brown, R., Higgins, P.J.: The equivalence of $\infty$-groupoids and crossed complexes. Cah. Top. Géom. Differ. 22, 371-386 (1981)

4. Brown, R., Higgins, P.J.: The classifying space of a crossed complex. Math. Proc. Cambr. Philos. Soc. 110, 95-120 (1991)

5. Breen, L.: Notes on 1- and 2-Gerbes. http://arxiv.org/abs/math/0611317 (2006)

6. Breen, L.: On the classification of 2-gerbes and 2-stacks. Soc. Math. de France 225 (1994)

7. Bousfield, A.K., Kan, D.: Homotopy limits, completions and localizations, Lecture Notes in Mathematics, vol. 304. Springer, Berlin (1972)

8. Dakin, M.K.: Kan complexes and multiple groupoid structures, Mathematical sketches, 32, Paper No. 2. Esquisses Math., 32, Univ. Amiens, Amiens (1983)

9. Dwyer, B., Farjoun, E.D.: A long homology localization tower. Comment. Math. Helvet. 52, 186-210 (1977)

10. Gray, J.W.: Formal category theory: adjointness for 2-categories, Lecture Notes in Mathematics, vol. 391. Springer, Berlin (1974)

11. Goerss, P., Jardine, R.: Simplicial homotopy theory, Progress in Mathematics, vol. 174. Birkhauser, Basel (1999)

12. Hirschhorn, P.S.: Model categories and their localizations, Mathematical Surveys and Monographs, vol. 99. AMS, Providence (2003)

13. Jardine, R.: Cosimplicial spaces and cocycles. http://www.math.uwo.ca/jardine/papers/preprints/ cosimp4.pdf (2010, preprint)

14. MacLane, S.: Categories for the Working Mathematician. Graduate Texts in Mathematics. Springer, Berlin (1971)

15. Moerdijk, I., Svensson, J.A.: Algebraic classification of equivariant homotopy 2-types, I. J. Pure Appl. Algebra 89, 187-216 (1993)

16. Noohi, B.: Notes on 2-groupoids, 2-groups and crossed modules. Homol. Homotopy Appl. 9(1), 75106 (electronic) (2007)

17. Quillen, D.G.: Homotopical algebra, Lecture Notes in Mathematics, vol. 43. Springer, New York (1967)

18. Reedy, C.L.: Homotopy theory of model categories, unpublished manuscript. http://www-math.mit. edu/psh/reedy.pdf 
19. Street, R.: The algebra of oriented simplexes. J. Pure Appl. Algebra 49(3), 283-335 (1987)

20. Yekutieli, A.: Combinatorial descent data for gerbes. http://arxiv.org/abs/1109.1919

21. Yekutieli, A.: MC elements in pronilpotent dg Lie algebras. J. Pure Appl. Algebra 216, 2338-2360 (2012) 